\title{
Neurophysiological characteristics in argininemia: a case report
}

\author{
Bin Cui ${ }^{1,2,3} \wedge$, Lin Wei ${ }^{1,2,3} \wedge$, Zhi-Jun Zhu ${ }^{1,2,3} \wedge$, Li-Ying Sun ${ }^{1,2,3,4} \wedge$ \\ ${ }^{1}$ Liver Transplantation Center, Beijing Friendship Hospital, Capital Medical University, Beijing, China; ${ }^{2}$ Clinical Center for Pediatric Liver \\ Transplantation, Capital Medical University, Beijing, China; ${ }^{3}$ National Clinical Research Center for Digestive Diseases, Beijing, China; ${ }^{4}$ Intensive \\ Care Unit, Beijing Friendship Hospital, Capital Medical University, Beijing, China \\ Correspondence to: Zhi-Jun Zhu, MD, PhD. Liver Transplantation Center, Clinical Center for Pediatric Liver Transplantation, National Clinical \\ Research Center for Digestive Diseases, Beijing Friendship Hospital, Capital Medical University, No. 95 Yong-an Road, Xi Cheng District, Beijing \\ 100050, China. Email: zhu-zhijun@outlook.com.
}

\begin{abstract}
Argininemia is a rare inherited disorder characterized by progressive spastic paraplegia, leading by mutation of the ARG1 gene. Liver transplantation (LT) had been reported to prevent symptoms progression, while its pathophysiology is still unclear. A 13-year-old male patient with argininemia for progressive neurological impairment was admitted to our center. Plasma amino acid screening showed a high concentration of arginine, and gene sequencing showed heterozygous mutation of the ARG1 gene. Spastic Paraplegia Rating Scale (SPRS), motor evoked potentials (MEPs), somatosensory evoked potentials (SEPs), F-wave, electromyography, nerve conduction velocity (NCV), and brain MRI were used to evaluate the patient. Herein, we describe the clinical characteristics of this patient, attempting a correlation between clinical, neurophysiological, and neuroimaging data in argininemia. Pyramidal tract dysfunction of lower limbs affected him, while only MEPs showed abnormalities among all neurophysiological evaluations, and mild cerebellum atrophy was observed. He responded poorly to traditional treatment such as a protein restriction diet and sodium benzoate. The symptoms of speech disorder, irritability, and dyskinesia were gradually deteriorating, so living-donor LT (LDLT) was done to prevent the progression. The symptoms improved significantly six months after LT, and the spasticity severity score decreased $50 \%$. The findings suggest that LDLT is effective to argininemia, and the phenotypical similarities to other disorders that affect the urea cycle (HHH syndrome and pyrroline-5-carboxylate synthetase deficiency) suggest a common mechanism may contribute to maintaining the integrity of the corticospinal tract.
\end{abstract}

Keywords: Argininemia; neurophysiological assessment; hereditary spastic paraplegias; pyramidal tract; case report

Submitted Mar 21, 2021. Accepted for publication May 27, 2021.

doi: $10.21037 / \mathrm{tp}-21-112$

View this article at: https://dx.doi.org/10.21037/tp-21-112

\section{Introduction}

Argininemia (arginase deficiency or hyperargininemia; OMIM 207800) is an autosomal recessive inherited disease caused by the deficiency of arginase 1 , which is a rare type of urea cycle disorder (UCD) (1). The gradual loss of mental and motor abilities, increasing severity of spasticity, and symptoms of pyramidal tracts are hallmarks of the disease. The pathophysiology of pyramidal tract dysfunction remains to be clarified. Brain magnetic resonance imaging (MRI) shows a variable degree of cerebral and cerebellum atrophy, while no metabolic abnormality was observed in a magnetic resonance spectroscopy study (2). Neurophysiological assessments such as motor evoked potentials (MEPs), somatosensory evoked potentials (SEPs),

^ ORCID: Bin Cui, 0000-0001-9941-5991; Lin Wei, 0000-0002-0435-3829; Zhijun Zhu, 0000-0001-7031-2083; Li-Ying Sun, 0000-00031101-7994. 


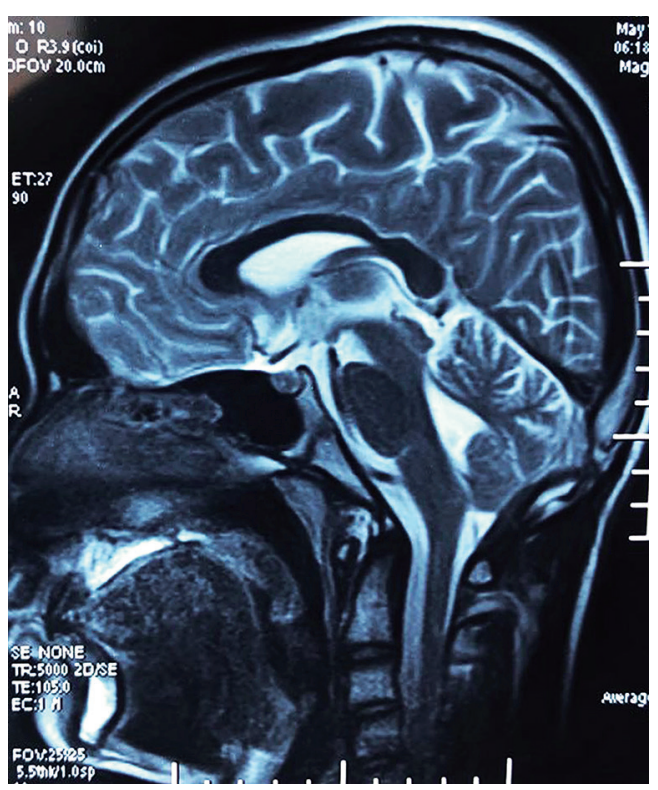

Figure 1 Mild cerebellar atrophy is observed in the sagittal T2-weighted image.

nerve conduction velocity ( $\mathrm{NCV}$ ), and F-wave provide a non-invasive objective method to evaluate both the central and peripheral nervous system, which is valuable in infants and young children $(3,4)$.

To our knowledge, neurophysiological evaluation in patients with argininemia has rarely been reported. Liver transplantation (LT) has been suggested to prevent progressive neurological impairment in argininemia (5). It is lucky for us to manage an argininemia patient who needed LT to improve the symptoms. Herein, we reported the neurologic, neurophysiological, clinical, and neuroimaging findings of argininemia to elucidate the pathophysiology and evaluate the effect of LT.

We present the following case in accordance with the CARE reporting checklist (available at https://dx.doi. org/10.21037/tp-21-112).

\section{Case presentation}

A 13-year-old male patient was admitted to our hospital. Spastic paraplegia, weakness of bilateral lower limbs, and tip-toeing gait were observed on admission. Protein aversion appeared at the age of 5 years old without any other symptoms. The patient developed dragging of the right lower limb four years ago, which was progressive to spastic paraplegia of both lower limbs recently. The patient showed significant social dysfunction, emotional instability, irritability, poor appetite, slow speech with discontinuity. His height was $175 \mathrm{~cm}$. It was difficult for him to bend down and stand up independently. Neurological examination revealed pyramidal tract signs in bilateral lower limbs, spastic gait abnormality, hyperreflexia of knee and ankle reflex, hypermyotonia, positive ankle clonus and Babinski signs, strength decreased to grade IV, while the upper limbs were unaffected. His spasticity severity score was 38/54 as assessed by the Spastic Paraplegia Rating Scale (SPRS) (6). Complex hereditary spastic paraplegias (HSPs) were diagnosed at first. The diagnosis of argininemia was confirmed by plasma amino acids analysis and gene sequencing. Blood arginine concentration was $150.9 \mu \mathrm{mol} / \mathrm{L}$ (reference range, $5-25 \mu \mathrm{mol} / \mathrm{L}$ ) on the first examination and reached a maximum of $289.1 \mu \mathrm{mol} / \mathrm{L}$. The likely pathogenic mutation, c.647T>C (p.L216P) and pathogenic mutation, c.263-266delAGAA (p.k88Rfs*45), were identified in the $A R G 1$ gene from his genomic DNA. His younger sister showed without any symptoms. Laboratory examination showed mild coagulation disorder without bleeding symptoms. Brain MRI revealed mild cerebral atrophy (Figure 1). The patient responded poorly to dietary management consisting of protein restriction, sodium benzoate, and sodium phenylbutyrate therapy. He could not walk or stand independently before surgery, just one month after admission.

\section{Neurophysiological evaluation}

Assessment of long fiber tracts included MEPs of the upper and lower limb corticospinal tracts and SEPs of lower limb afferent somatosensory tracts. NCV and F-wave evaluated the peripheral nervous system. Motor NCV was recorded at the tibial nerve, common peroneal nerve, median nerve, ulnar nerve, and sensory NCV at the superficial peroneal nerve, median nerve, sural nerve, and ulnar nerve. F-wave was recorded at the tibial nerve. Electromyography (EMG) signals were recorded at the surface of the tibialis anterior muscle, gastrocnemius muscle, and quadriceps femoris muscle. Only tibial SEPs were recorded, and recordings in response to stimulation of the posterior tibial muscle were obtained from the contralateral cortex $\mathrm{Cz}(2 \mathrm{~cm}$ posterior to the $\mathrm{Cz})$, referred to as Fz. The components P40, N50, P60, and N75, were evaluated. MEPs were performed by stimulating the motor cortex with repetitive transcranial magnetic stimulation and recorded at the abductor digiti minimi muscle for the upper limbs and at the tibialis anterior muscle for the lower 
Table 1 The PMCT, latency of MEPs, and CMCT of bilateral limbs pre-operation

\begin{tabular}{|c|c|c|c|c|c|c|}
\hline & \multicolumn{3}{|c|}{ Upper limb } & \multicolumn{3}{|c|}{ Lower limb } \\
\hline Left & 13.5 & 24.1 & 10.6 & 14.0 & 42.3 & 28.3 \\
\hline Right & 13.6 & 23.5 & 9.9 & 15.0 & 41.7 & 26.7 \\
\hline
\end{tabular}

PMCT, peripheral motor conduction time; MEPs, motor evoked potentials; CMCT, central motor conduction time.

limbs during relaxation. The central motor conduction time (CMCT) at the target muscle was estimated by subtracting the conduction time from the spinal roots to the muscle and referred to as the peripheral motor conduction time (PMCT) from the latency of MEPs. The center of the magnetic coil was placed over the C7 cervical spine or L4 lumbar spine to stimulate the lower segment's spinal nerve roots to acquire the PMCT. CMCT values of lower limbs were considered abnormal if $>20 \mathrm{~ms}$ or with a side-to-side difference $>2 \mathrm{~ms}$ (3). The other indicators were considered abnormal if $>$ mean \pm 2.5SD. The reference ranges were obtained from literature data and studies performed at our hospital on healthy agematched pediatric subjects.

All procedures performed in studies involving human participants were in accordance with the ethical standards of the institutional and/or national research committee(s) and with the Helsinki Declaration (as revised in 2013). Consent for publication was obtained from the guardian of the participant. This study was approved by the Ethics Committee of Beijing Friendship Hospital, Capital Medical University (2020-P2-094-01).

\section{Results}

For the scarcity of deceased organs, living-donor LT (LDLT) was performed after the comprehensive preoperative evaluation. His father was heterozygote as the donor, and the operation was approved by the Ethics Committee of Beijing Friendship Hospital, Capital Medical University. We followed the patient after operation every one or two weeks within three months, then every one month within six months. Although the plasma arginine gradually decreased to an average value six months after the operation, he regained language expression and speech fluency without any social dysfunction within one month. He manifested an excellent appetite and gained $2.5 \mathrm{~kg}$ until now. The symptoms of irritability disappeared one-week post-transplant. The knee-jerk reflex returned to normal. The muscle tension of the lower extremity improved significantly at rest, and he could bend down easily; however, gastrocnemii showed hypermyotonia when activity, and the ankles clonus without any improvement. The spasticity severity score decreased to 19/54. Although not all symptoms were improved, he can walk independently for 300 meters without rest or any assists. No complications of the operation affected him.

The data on neurophysiological assessments were obtained two months before the operation. Interestingly, the values of motor and sensory $\mathrm{NCV}$ were within normal, and the occurrence rates and latency of F-wave showed no abnormalities. EMG signals indicated that the muscles of the lower limbs were not affected. The latency of SEPs was within normal ranges in bilateral lower limbs. However, prolonged latency of MEPs was observed in bilateral upper and lower limbs, while the PMCT was normal. The CMCT of the lower limbs was 28.3 and $26.7 \mathrm{~ms}$ on the left and right side, respectively. The data were listed in Table 1. Until now, we did not perform the second neurophysiological evaluation after the operation.

\section{Discussion}

The spastic severity score evaluated by SPRS decreased significantly six months after the surgery. Although the symptoms were not entirely reversed within a short time after the operation, significant improvement suggested that LDLT could prevent aggravation of symptoms $(5,7,8)$.

In addition to protein aversion and language impairment, spastic paraplegia was the primary symptom, which was consistent with previous reports $(1,9)$. The neurophysiological evaluation suggested that the corticospinal tract was primarily damaged. A reduction in diffusion tensor imaging fiber count was observed in a patient with argininemia (10), which indicates that subcortical white matter below the motor cortex may display significant alterations and explain the presence of brain atrophy (2).

Spastic paraplegia of the lower limbs, cerebellar signs, and intellectual developmental disability highlight the 
importance of argininemia being listed as HSPs (11). Compared with the ascending sensory tract, this patient's neurophysiological data confirmed that descending motor tracts were mainly involved in argininemia. In contrast, the peripheral nerves and muscles were not involved. These findings of this patient are consistent with the research on Hyperornithinemia-hyperammonemia-homocitrullinuria (HHH) syndrome (3). Clinical, neurophysiological, and neuroradiological findings revealed similarities between argininemia, HHH syndrome, and delta-1-pyrrolin5 -carboxylate synthase (P5CS) deficiency. The results indicating a common mechanism related to the impairment of the corticospinal tract is involved in these diseases. In the urea metabolism cycle, arginase deficiency leads to decreased ornithine concentration in the mitochondrial pool, which is a common feature in argininemia, HHH syndrome, and P5CS deficiency (12), and it may be responsible for the common phenotypes. According to a recent study (10), dysmyelination caused by oligodendrocyte injury is the pathological characteristic of argininemia, CMCT and MEP latency are specifically associated with the maturation of the corticospinal tracts (10). Abnormal mitochondrial enlargement and decreased production of adenosine triphosphate (ATP) due to arginase gene mutations have been observed in model of argininemia (13). Taken together, mitochondrial ornithine deficiency may be responsible for oligodendrocyte degeneration and corticospinal tract immaturity in these diseases. And further research should investigate the theory. SEPs have been shown to be valuable for prognosis after brain injury in previous reports $(14,15)$, as sensory input and integration are important in learning motor skills, suggesting that our patient may have a good prognosis.

Of course, heterogeneity of neurophysiological data could exist in argininemia. However, the findings in this patient provide clues for further elucidation of the mechanism of argininemia. In the future, the neurophysiological assessment will likely be applied in argininemia patients frequently.

\section{Conclusions}

Our finding highlights the involvement of the corticospinal tract in argininemia; meanwhile, sensory tracts and peripheral nerves are not involved. The results of this patient suggest that argininemia should be listed in complex HSPs. LDLT is an effective strategy for preventing neurological impairment progression in argininemia.

\section{Acknowledgments}

We sincerely thank the family members and patient who participated in this study.

Funding: This work was supported by Capital's Funds for Health Improvement and Research (No.2020-1-2024); National Natural Science Foundation of China (No. 81970562)

\section{Footnote}

Reporting Checklist: The authors have completed the CARE reporting checklist. Available at https://dx.doi. org/10.21037/tp-21-112

Conflicts of Interest: All authors have completed the ICMJE uniform disclosure form (available at https://dx.doi. org/10.21037/tp-21-112). The authors have no conflicts of interest to declare.

Ethical Statement: The authors are accountable for all aspects of the work in ensuring that questions related to the accuracy or integrity of any part of the work are appropriately investigated and resolved. All procedures performed in studies involving human participants were in accordance with the ethical standards of the institutional and/or national research committee(s) and with the Helsinki Declaration (as revised in 2013). This study was approved by the Ethics Committee of Beijing Friendship Hospital, Capital Medical University (2020-P2-094-01). Consent for publication was obtained from the guardian of the participant.

Open Access Statement: This is an Open Access article distributed in accordance with the Creative Commons Attribution-NonCommercial-NoDerivs 4.0 International License (CC BY-NC-ND 4.0), which permits the noncommercial replication and distribution of the article with the strict proviso that no changes or edits are made and the original work is properly cited (including links to both the formal publication through the relevant DOI and the license). See: https://creativecommons.org/licenses/by-nc-nd/4.0/.

\section{References}

1. Sin YY, Baron G, Schulze A, et al. Arginase-1 deficiency. J Mol Med (Berl) 2015;93:1287-96.

2. Carvalho DR, Farage L, Martins BJ, et al. Brain MRI and 
magnetic resonance spectroscopy findings in patients with hyperargininemia. J Neuroimaging 2014;24:155-60.

3. Olivieri G, Pro S, Diodato D, et al. Corticospinal tract damage in $\mathrm{HHH}$ syndrome: a metabolic cause of hereditary spastic paraplegia. Orphanet J Rare Dis 2019;14:208.

4. Rossini PM, Burke D, Chen R, et al. Non-invasive electrical and magnetic stimulation of the brain, spinal cord, roots and peripheral nerves: Basic principles and procedures for routine clinical and research application. An updated report from an I.F.C.N. Committee. Clin Neurophysiol 2015;126:1071-107.

5. Kido J, Matsumoto S, Mitsubuchi H, et al. Early liver transplantation in neonatal-onset and moderate urea cycle disorders may lead to normal neurodevelopment. Metab Brain Dis 2018;33:1517-23.

6. Schüle R, Holland-Letz T, Klimpe S, et al. The Spastic Paraplegia Rating Scale (SPRS): a reliable and valid measure of disease severity. Neurology 2006;67:430-4.

7. Zhu ZJ, Wei L, Qu W, et al. First case of cross-auxiliary double domino donor liver transplantation. World J Gastroenterol 2017;23:7939-44.

8. Silva ES, Cardoso ML, Vilarinho L, et al. Liver transplantation prevents progressive neurological impairment in argininemia. JIMD Rep 2013;11:25-30.

Cite this article as: Cui B, Wei L, Zhu ZJ, Sun LY. Neurophysiological characteristics in argininemia: a case report. Transl Pediatr 2021;10(7):1947-1951. doi: 10.21037/tp-21-112
9. Lee BH, Jin HY, Kim GH, et al. Argininemia presenting with progressive spastic diplegia. Pediatr Neurol 2011;44:218-20.

10. Liu XB, Haney JR, Cantero G, et al. Hepatic arginase deficiency fosters dysmyelination during postnatal CNS development. JCI Insight 2019; 4:e130260.

11. Panza E, Martinelli D, Magini P, et al. Hereditary Spastic Paraplegia Is a Common Phenotypic Finding in ARG1 Deficiency, P5CS Deficiency and HHH Syndrome: Three Inborn Errors of Metabolism Caused by Alteration of an Interconnected Pathway of Glutamate and Urea Cycle Metabolism. Front Neurol 2019;10:131.

12. Coutelier M, Goizet C, Durr A, et al. Alteration of ornithine metabolism leads to dominant and recessive hereditary spastic paraplegia. Brain 2015;138:2191-205.

13. Tang R, Wang X, Zhou J, et al. Defective arginine metabolism impairs mitochondrial homeostasis in Caenorhabditiselegans. J Genet Genomics 2020;47:145-56.

14. Sleigh JW, Havill JH, Frith R, et al. Somatosensory evoked potentials in severe traumatic brain injury: a blinded study. J Neurosurg 1999;91:577-80.

15. White CP, Cooke RW. Somatosensory evoked potentials following posterior tibial nerve stimulation predict later motor outcome. Dev Med Child Neurol 1994;36:34-40. 